\title{
Dielectric Behaviour of MgZn W-type Hexaferrite
}

\author{
D.El Kony, S.A. Saafan, A.M. Abo El Ata.
}

Physics Department, Faculty of Science, Tanta Univ., Tanta, Egypt.

A series of polycrystalline $\mathrm{Ba} \mathrm{Zn}_{2-x} \mathrm{Mg}_{x} \mathrm{Fe}_{16} \mathrm{O}_{27}$ W-type hexagonal ferrite; $(x=0.0,0.4,0.8,1.2,1.6$ and 2$)$ were prepared by using the conventional ceramic method. The dielectric constant $\left(\varepsilon^{\prime}\right)$ and the dielectric loss tangent $(\tan \delta$ ) were determined as functions of frequency, temperature and composition. The frequency dependence of the dielectric constant was found to exhibit two types of behaviour: the usual dispersion at low temperatures and an abnormal behaviour at relatively high temperatures which could be explained on the basis of Rezlescu and Rezlescu assumption, which attributes this abnormal behaviour to a collective contribution of two types of charge carriers to the polarization. 


\section{Introduction}

Today ceramic magnetic materials, capable of combining a high resistivity and a high permeability, are found in numerous products used in our every day life such as home appliances, electronic devices, communication equipments and computers [1]. Ferrimagnetic materials can have one of three main structures; spinel, garnet, and hexagonal [2]. For the hexagonal structure, there are six possible different types designated $\mathrm{M}, \mathrm{W}, \mathrm{Y}, \mathrm{Z}, \mathrm{X}$, and $\mathrm{U}$. the $\mathrm{W}$-type hexagonal ferrites $\mathrm{Ba} \mathrm{Me} \mathrm{Fe}_{16} \mathrm{O}_{27}$, (where Me stands for any divalent element), have a crystalline structure built up as a superposition of $\mathrm{S}$ and $\mathrm{R}$ blocks, where the $\mathrm{S}$ block has the formula $\mathrm{Fe}_{6} \mathrm{O}_{8}$ and $\mathrm{R}$ block has the formula $\mathrm{Ba} \mathrm{Fe}_{6} \mathrm{O}_{11}$. The unit cell is composed of the sequence R S S R* $\mathrm{S}^{*} \mathrm{~S}^{*}$. Between $\mathrm{S}$ and $\mathrm{S}^{*}$, and between $R^{*}$, a $180^{\circ}$ rotation about the c-axis occurs [3]. However, the presence of divalent and trivalent cations distributed among various sublattices makes the W-type very interesting for basic studies and different technical applications, since their characteristics may, in principle, be varied by substitution of both divalent and trivalent cations.

\section{Experimental Techniques}

A series of polycrystalline $\mathrm{Ba}$ hexagonal ferrite with composition formula $\mathrm{Ba} \mathrm{Zn}_{2-\mathrm{x}} \mathrm{Mg}_{\mathrm{x}} \mathrm{Fe}_{16} \mathrm{O}_{27}$ (where $\mathrm{x}=0.0,0.4,0.8,1.2,1.6$ and 2) was prepared by the conventional ceramic technique where a mixture in the proper proportions of highly pure (more than $99 \%$ ) $\mathrm{Ba} \mathrm{CO}_{3}, \mathrm{MgO}, \mathrm{ZnO}$, andFe $\mathrm{O}_{3}$ were ground in an electrical grinding machine for ten hours. Then the resulted fine powders were presintered in air in an electric furnace at $900^{\circ} \mathrm{C}$ for 20 hours, and were slowly cooled within 48 hours to room temperature. The grinding process was repeated again, afterwards the powders were pressed into disk-shaped pellets with diameters ranging from $(1.4-1.5 \mathrm{~cm})$ and thicknesses ranging from $(0.2-0.4 \mathrm{~cm})$. The pressed samples were finally sintered in air at $1250^{\circ} \mathrm{C}$ for 20 hours and left to be slowly cooled again by reducing the temperature by $50^{\circ} \mathrm{C}$ every two hours until reaching $700^{\circ} \mathrm{C}$, then the furnace was switched off and the samples were left to be cooled to room temperature.

X-ray diffraction analysis confirmed the presence of a single-phase $\mathrm{W}$-type hexagonal ferrite. The samples were polished and coated by a thin layer of silver paste as a good contact material for electrical measurements. The dielectric and ac conductivity measurements were performed using the couple impedance technique (a lock-in amplifier: SR 510 Stanford Research Systems). A Block diagram of the circuit used is mentioned in a previously published work [4]. 


\section{Results and discussion \\ Dielectric constant $\left(\boldsymbol{\varepsilon}^{\prime}\right)$}

Figure (1) shows the frequency dependence of the dielectric constant for the six prepared samples. We can see obviously two types of behaviour; the first, observed at low temperatures, is a traditional dispersion or decrease of the dielectric constant with increasing frequency, and this behaviour is always attributed to the lagging of the existing charge carriers - (hopping electrons between $\mathrm{Fe}^{2+}$ and $\mathrm{Fe}^{3+}$ ions (at localised sites); where the $\mathrm{Fe}^{2+}$ were formed in the samples during the sintering process at elevated temperature [5])responsible for polarization behind the applied field as its frequency increases $[6,7,8]$. The second behaviour was observed at relatively high temperatures, where the dielectric constant at first, increases with increasing frequency until
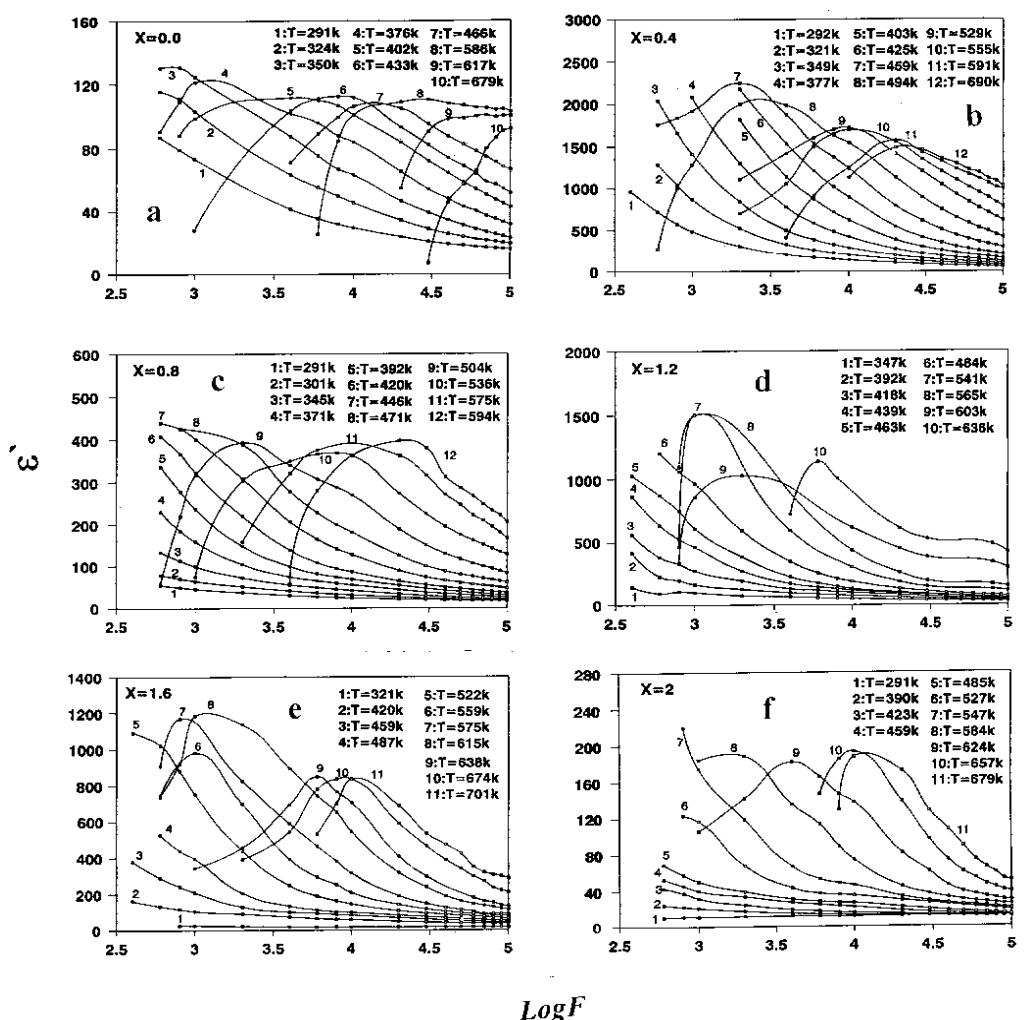

Fig. (1) : Frequency dependence of the dielectric constant at different temperatures for the six investigated compositions.

reaching a peak after which it displays the traditional relaxation, this behaviour can be explained on the basis of Rezlescu and Rezlescu assumption [9], where this hehaviour was attributed to a collective contribution of electrons and holes to the polarization. In our samples, this assumption can be applied since Ghani 
et al. [10], suggested the existence of donor and acceptor centers in the lattice of the ferrite. The formation of both types of centers arises from the loss of oxygen during the sintering process. For charge compensation a part of $\mathrm{Fe}^{3+}$ ion transforms to $\mathrm{Fe}^{2+}$ ions which act as donor centers. Moreover, the loss of oxygen may cause a part of cations to occupy interstitial sites and act as acceptor centers .Moreover, the peak appearing in the dielectric constant versus frequency curve, shifts towards higher frequencies with increasing temperature, and this is due to the increase of the thermally activated hopping frequency of the charge carriers between localised sites [9].

From the same figures, we can see that, generally the dielectric constant values are high particularly at low frequencies, this can be attributed to the interfacial polarization known to occur in heterogenous structures $[6,11]$.

Figure (2) shows the temperature dependence of the dielectric constant for all samples at five selected frequencies. It can be seen that the dielectric constant increases with increasing temperature, -as expected for the semiconducting behaviour of most ferrite systems - , until reaching a maximum, then decreases with further increase in temperature. Moreover, the maximum value shifts towards higher temperatures with increasing frequency. This behaviour can be attributed to the presence of two types of charge carriers as mentioned above and since the mobility of holes is smaller than that of electrons, therefore, it is expected that their contribution to polarization appears only at high temperatures causing that observed decrease as a result of two contributions with opposite signs.

Figure (3) shows the $\log$ of the dielectric relaxation frequency (at which the peak of $\varepsilon^{\prime}$ vs. $\log \mathrm{F}$ curve appears) as a function of $10^{3} / \mathrm{T}$ for all compositions. The plotted lines verify the equation [12]:

$$
f_{d}=f_{o} \exp \left[-E_{d} / k T\right],
$$

where $f_{o}$ is a pre exponential constant, giving straight line for each sample and indicating the presence of a well defined activation energy also. The activation energy for each line was calculated and given in Table (1). It is seen that $E_{d}$ is not systematically varied with composition. 

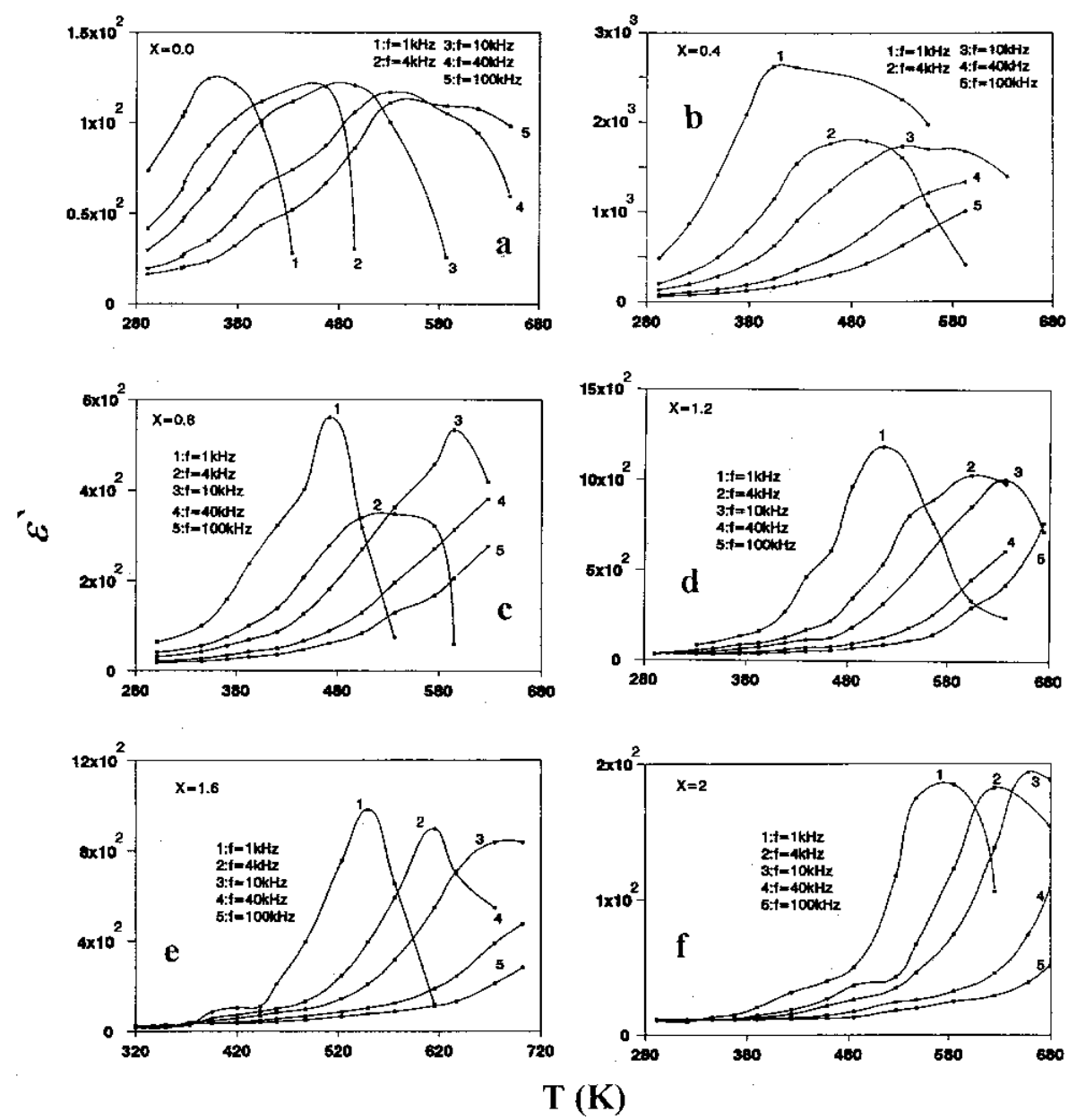

Fig. (2): Temperature dependence of the dielectric constant at different frequencies for the six investigated compositions.

Table (1):The activation energy for the investigated compositions

\begin{tabular}{|c|c|c|c|c|c|c|}
\hline$\underline{\mathrm{X}}$ & 0.0 & 0.4 & 0.8 & 1.2 & 1.6 & 2 \\
\hline $\mathrm{E}_{\mathrm{d}}(\mathrm{EV})$ & 0.264 & 0.399 & 0.663 & 0.412 & 0.623 & 0.566 \\
\hline
\end{tabular}




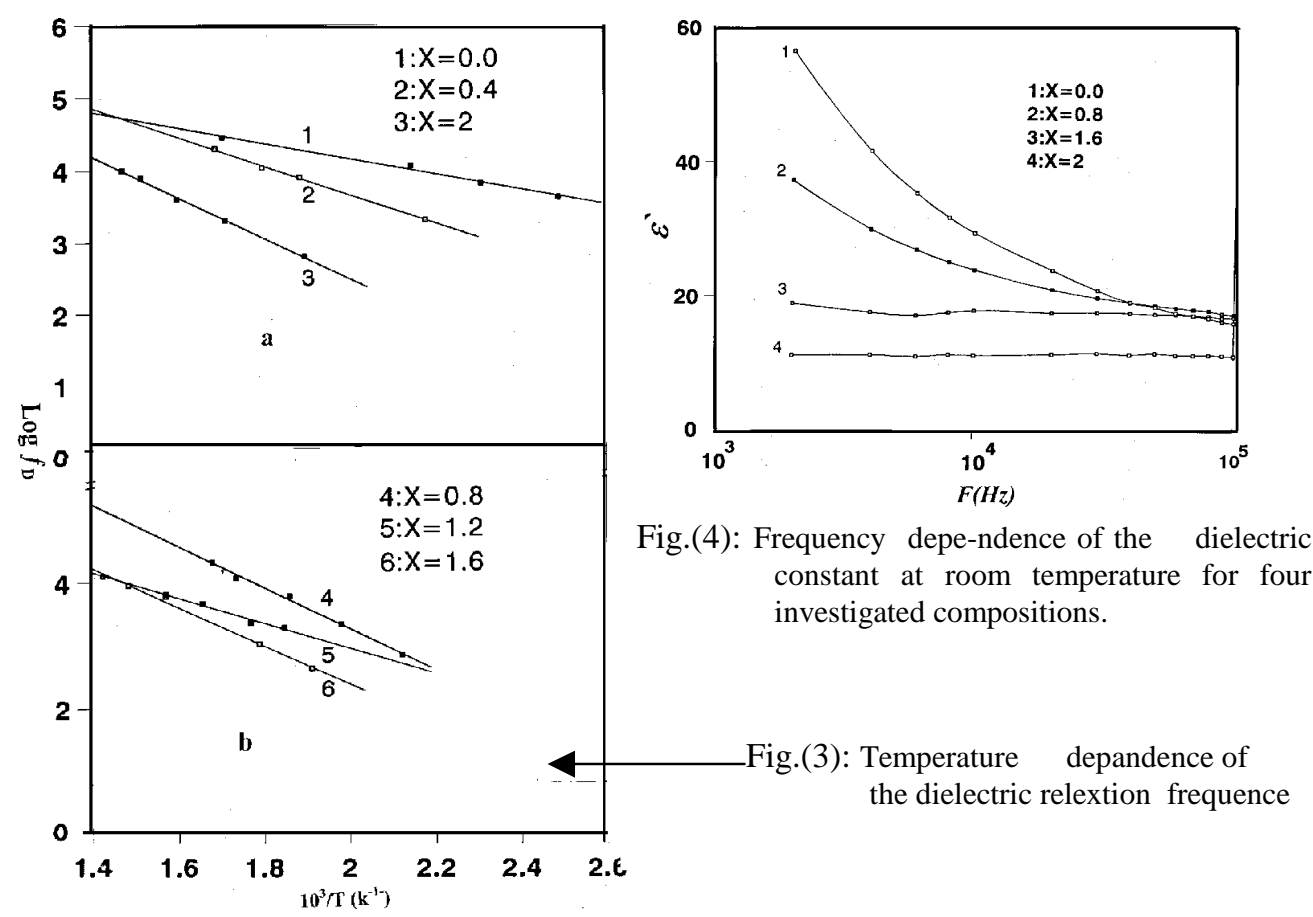

Figure (4) represents the frequency dependence of the dielectric constant at room temperature for different compositions. It can be obviously noticed that the sample of $\mathrm{x}=2$ (maximum Mg content) shows no frequency dependence at room temperature besides it has a relatively low value of $\varepsilon^{\prime}$. In other words by increasing $\mathrm{Mg}$ content the dielectric constant decreases. This behaviour can be explained as follows: The $\mathrm{Zn}^{2+}$ ion shows a marked preference of tetrahedral sites, where its $4 \mathrm{~s}$ and $\mathrm{p}$ electrons can form a covalent bond with the six $2 p$ electrons of the oxygen ion in spinel ferrites [13]. This was taken as a general rule, where the nonmagnetic zinc ions occupy tetrahedral coordinationinin $\mathrm{Zn}_{2} \mathrm{~W}$-ferrite as in spinel ferrites. The cation distribution in $\mathrm{Mg}_{2}$ W-ferrite deduced from NMR and Mossbauer spectra indicates that $\mathrm{Mg}$ ions mainly occupy the octahedral sites inside the S-blocks [14]. Therefore, the substitution of $\mathrm{Zn}$ ions by $\mathrm{Mg}$ ions forces some ferric ions to migrate to the tetrahedral sites causing a decrease of the hopping charge carriers in octahedral sites which consequently leads to a decrease in polarizatin and dielectric constant values. 

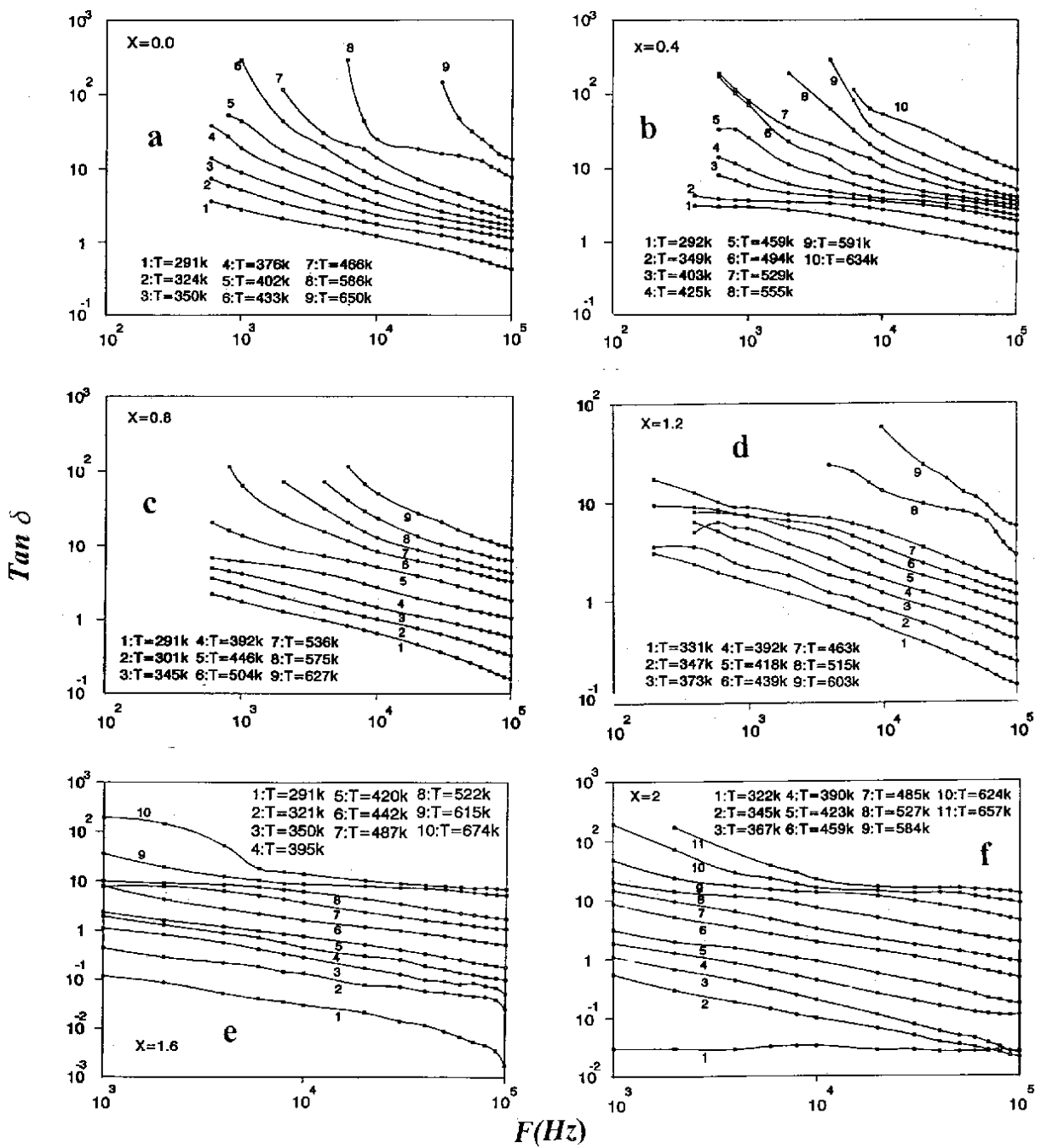

Fig. (5): Frequency dependence of loss tangent at differenet temperatures.

\section{Dielectric losses $(\tan \delta)$}

Figure (5) shows the loss tangent behaviour as a function of frequency at different temperatures for all samples. The loss tangent $(\tan \delta)$ is defined as the ratio of the loss or resistive current to the charging current in the sample. Also it is known that, there is a strong correlation between the conduction mechanism and the dielectric constant behaviour (the polarization mechanism) in ferrites. From these two considerations we can see that he behaviour of $\tan \delta$ with frequency is showing the expected decrease of $\tan \delta$ with increasing frequency. Also, the increase in $\tan \delta$ value (i.e. in loss current) with increasing 
temperature ensures the semiconducting nature or the thermally activated mechanism of conduction in the samples.

Figure (6) shows the temperature dependence of $\tan \delta$ at five selected frequencies for all samples. We can notice that the general tendency of the curves is the same as that of the dielectric constant in agreement with the previously mentioned correlation between polarization and conduction mechanisms. Moreover, the shoulder observed to shift to higher frequencies with increasing temperature ensures the assumption of the presence of two types of charge carriers.
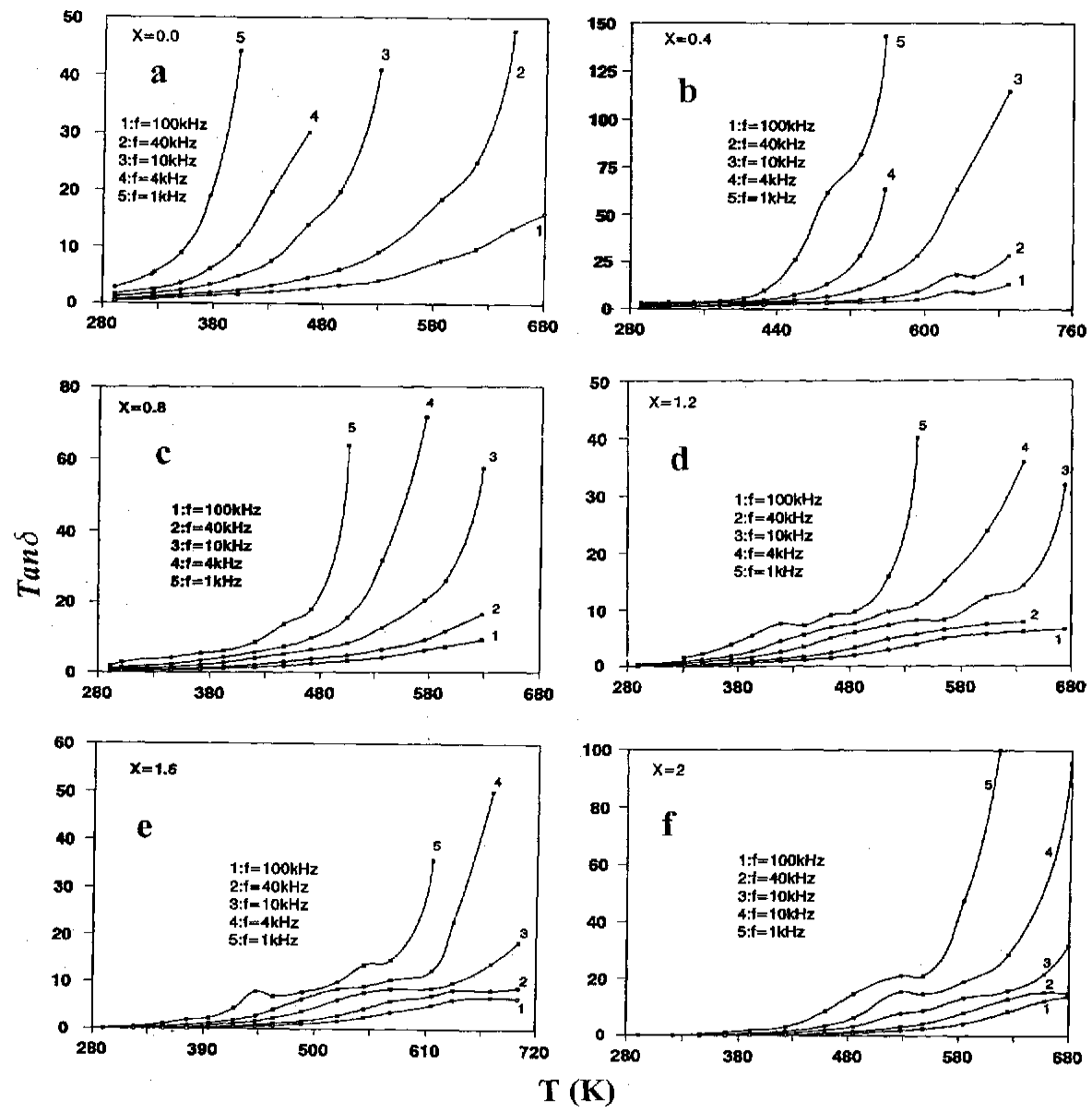

Fig. (6) : Temperature dependence of loss tangent at different frequencies.

Finally, the similarity of Fig.(7), which displays the frequency dependence of $\tan \delta$ for different compositions at room temperature, to Fig. (4) ensures the above considerations and assumptions. 


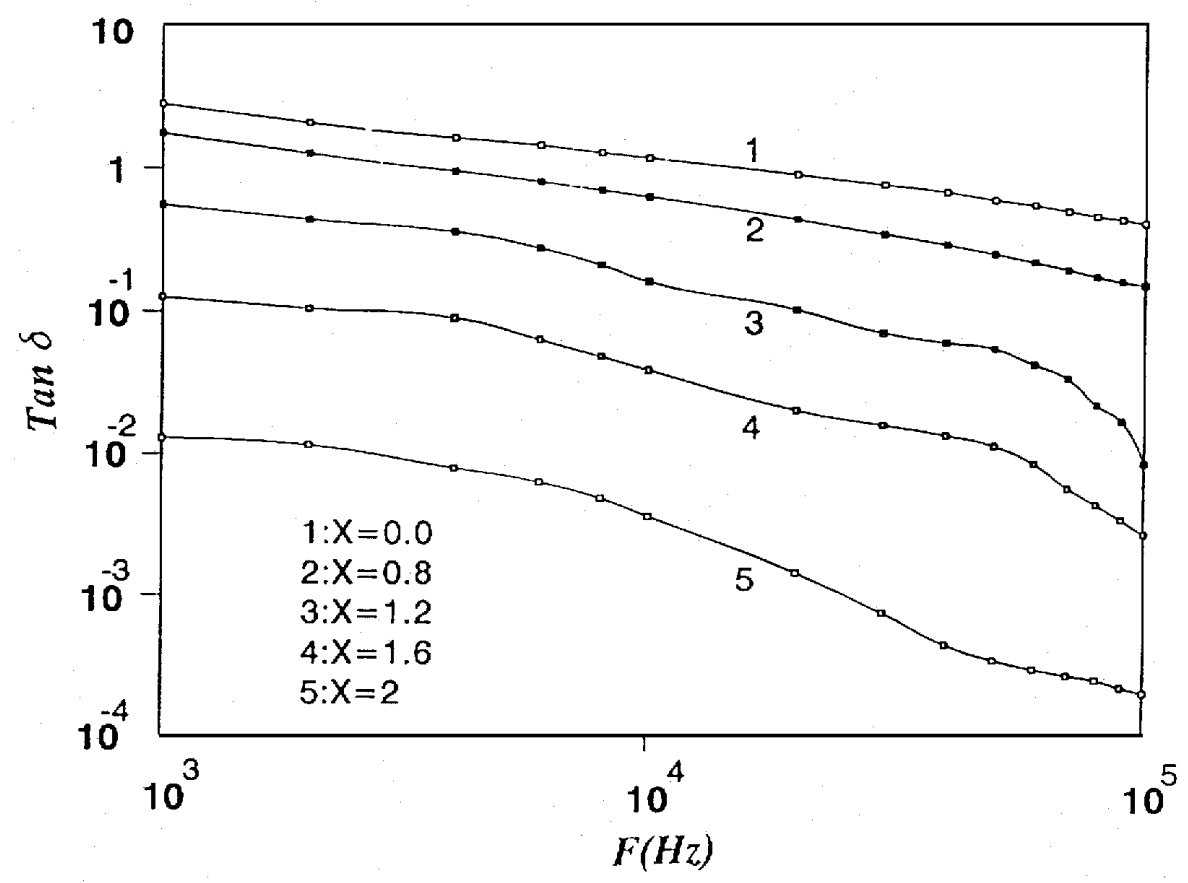

Fig. (7) : Frequency dependence of loss tangent at room temperature for four investigated compositions.

\section{Conclusions}

The main conclusions that can be drawn out from the above results are:

(1) The abnormal behaviour of the dielectric constant as a function of frequency at relatively high temperatures results from a collective contribution to the polarization process from two types of charge carriers.

(2) The existence of gradually shifted shoulders - in the $\tan \delta$ vs. temperature curves - towards higher frequencies ensures also the presence of two types of charge carriers.

(3) The relatively high values of the dielectric constant at low frequencies are due to the interfacial polarization ensuring that the samples can be fairly considered as formed of well conducting grains and poorly conducting grain boundaries in agreement with Koops model.

\section{Acknowledgment}

The authors would like to express their thanks and gratitude to professor Dr.M.K. El Nimr, Head of Solid state Physics and Electronics Lab., Faculty of Secience, Tanta University, for his constant support and helpful advice. 


\section{References}

1. B.Viswanathan and V.R.K. Murthy, Ferrite Materials: Science and Technology (Narosa publishing house, New Delhi, 1990), p.429.

2. W.H. Von Aulock and A.H. Bobeck, Ferrimagnestism: The Encyclopedia of Physics, third edition, Robert M.Besancon, Van Nostrand Reinold, New York, 1990), p. 429.

3. K.J. Standley, Oxide Magnetic Materials (Clarendon Press, Oxford, 1962), p.36.

4. A.M. Abo El Ata and M.A. El Hiti, J. Phys. III France 7 (1997) 883.

5. C. Parakash, J.Mater. Sci. Lett. 6 (1987) 651.

6. A.A. Zaky and R.Hawley, Dielectric Solids (Routledge \& Kegan Paul Ltd., London, 1970) p.21,27.

7. V.R. Murthy and J.Sobhanadri, phys. stat. sol. (a) 36 (1977) K133.

8. P.V. Reddy and T.S. Rao, J.Less Comm. Metals 86 (1982) 255.

9. N.Rezlescu and E.Rezlescu, Phys. Stat. Sol. (a) 23, (1974) 575.

10. A.A. Ghani, A.I. Eatah and A.A. Mohamed, Proc. Inter. conf. Japan, Sept.Oct. (1980) 216.

11. C.G. Koops, phys. Rev. 83 (1951) 121.

12. A. Mansingh, V.K. Dhawain and H.Sayer, Philos. Mag. (B) 48 (1983) 215.

13. J. Smit and H.P.J. Wijn, Ferrites (Philips Technical Library, Eindhoven, The Netherlands, 1959) p.142.

14. G.Albanese, J.Phys.Suppl., 38 C1 (1977) 85. 